\title{
COMPETÊNCIA DA ENFERMEIRA NO CUIDADO PRÉ-NATAL: POTENCIALIDADES, ENTRAVES E POSSIBILIDADES
}

\section{NURSING COMPETENCE IN PRENATAL CARE: POTENTIALITIES, BARRIERS AND POSSIBILITIES}

\section{COMPETENCIA DE LA ENFERMERA EN EL CUIDADO PRÉNATAL: POTENCIALIDADES, BARRERAS Y POSIBILIDADES}

Deisi Cristine Forlin Benedet ${ }^{1}$, Marilene Loewen Wall ${ }^{1}$, Maria Ribeiro Lacerda ${ }^{1}$, Andréa Cristina de Morais Chaves Thuler ${ }^{1}$, Camila Caroline Szpin ${ }^{1}$, Adriana Aparecida Piler ${ }^{1}$

\section{RESUMO}

Objetivo: Identificar potencialidades, entraves e possibilidades, quanto ao cuidado pré-natal com competência. Método: Revisão integrativa realizada, em junho de 2018, em cinco bases de dados de amplo acesso na área da saúde, combinaram-se descritores e termos relativos à temática. Incluíram-se artigos originais, disponíveis na íntegra, nos idiomas inglês, espanhol ou português, sem delimitação temporal. Resultados: Foram incluídos 20 estudos, 55\% de abordagem quantitativa, e 20\% apresentam a definição de competência utilizada. A escuta ativa, vínculo e orientações em saúde são potencialidades apontadas por gestantes e enfermeiras; déficit de conhecimento e habilidade, alta rotatividade de profissionais, metas quantitativas e excesso de trabalho burocrático foram alguns entraves destacados; e educação permanente, feedback da população e maior articulação serviço, ensino e associações de classe constituem-se em algumas possibilidades para desenvolvimento de competência no cuidado prénatal. Considerações finais: Para aperfeiçoar o cuidado prestado, exige-se um esforço coletivo, tanto pessoal como em equipe, das instituições de serviço, ensino e classe, para que a formação desenvolva a competência profissional e, em serviço, essa se perpetue direcionada à responder as necessidades de saúde das gestantes.

Descritores: Competência profissional; Cuidado Pré-Natal; Cuidado de Enfermagem; Atenção Primária à Saúde.

\begin{abstract}
Objective: To identify potentialities, barriers and possibilities regarding competently prenatal care. Method: Integrative review conducted in June 2018 in five widely accessible health databases by combining descriptors and terms related to the subject. Original articles, available in full in English, Spanish or Portuguese, without temporal delimitation were included. Results: Twenty studies were included, 55\% presenting a quantitative approach and $20 \%$ presenting the definition of competence. Active listening, bonding and health orientations are potentialities pointed out by pregnant women and nurses; lack of knowledge and skill, high staff renewal rate, quantitative goals and excessive bureaucratic work were some highlighted obstacles. Continuing education, population feedback and greater articulation service, teaching and class associations are some possibilities for developing prenatal care competence. Final considerations: To improve the provided care requires a collective effort, both personal and team, of the service, teaching and class institutions, so that the training develops the professional competence and in service that is perpetuated directed to answer the needs of health of pregnant women.
\end{abstract}

Descriptors: Professional Competence; Prenatal Care; Nursing Care; Primary Health Care.

\section{RESUMEN}

Objetivo: Identificar potencialidades, barreras y posibilidades con respecto a la atención prenatal competente. Método: Revisión integradora realizada en junio del 2018 en cinco bases de datos de amplio acceso en el área de la salud, se combinaron descriptores y términos de la temática. Se incluyeron artículos originales, en su totalidad, en inglés, español o portugués, sin delimitación temporal. Resultados: Se incluyeron 20 estudios, $55 \%$ de abordaje cuantitativo, y $20 \%$ presentan la definición de competencia utilizada. La escucha activa, el vínculo y las orientaciones de salud son potencialidades señaladas por mujeres embarazadas y enfermeras; los déficits de conocimiento y habilidades, la alta rotación de profesionales, los objetivos cuantitativos y el trabajo burocrático excesivo fueron algunos obstáculos destacados; y la educación continua, la retroalimentación de la población y un mayor servicio de articulación, enseñanza y asociaciones de clase son algunas posibilidades para desarrollar la competencia de atención prenatal. Consideraciones finales: Para mejorar la atención brindada se requiere un esfuerzo colectivo, tanto personal como de equipo, del servicio, instituciones de enseñanza y en clase, para que en la formación se desarrolle la competencia profesional y en el servicio para que se perpetúe en la dirección de responder a las necesidades de la salud de la embarazada.

Descriptores: Competencia Profesional; Atención Prenatal; Atención de Enfermería; Atención Primaria de Salud.

'Programa de Pós-Graduação em Enfermagem Universidade Federal do Paraná UFPR, Brasil. Membro do Núcleo de Estudos, Pesquisa e Extensão em Cuidado Humano de Enfermagem. ${ }^{2}$ Enfermeira. Professora do Departamento de Enfermagem da UFPR. Vice-líder do Núcleo de Estudos, Pesquisa e Extensão em Cuidado Humano e Enfermagem. ${ }^{3}$ Enfermeira. Professora do Programa de Pós-Graduação em Enfermagem da UFPR. Professora Visitante do Programa de Pós-Graduação de Enfermagem da UFSM. ${ }^{4}$ Doutoranda do Programa de Pós-Graduação em Enfermagem da UFPR. Membro do Núcleo de Estudos, Pesquisa e Extensão em Cuidado Humano e Enfermagem. ${ }^{5}$ Enfermeira no Hospital de Clínicas da UFPR. Mestranda do Programa de Mestrado Profissional em Enfermagem da UFPR. ${ }^{6}$ Enfermeira do Hospital de Clínicas da UFPR. Mestre em Enfermagem pelo Programa de Mestrado Profissional da UFPR.

\section{Como citar este artigo:}

Benedet DCF, Wall ML, Lacerda MR, et al. Competência da enfermeira no cuidado pré-natal: potencialidades, entraves e possibilidades.. Revista de Enfermagem do Centro-Oeste Mineiro. 2019;9e3470. [Acess http://dx.doi.org/10.19175/recom.v9i0.3544 


\section{INTRODUÇÃO}

A prestação de cuidados de alta qualidade à saúde da gestante incide, positivamente, para a redução das taxas de morbimortalidade materna e infantil. Entre as diversas práticas desenvolvidas pela enfermeira na Atenção Primária à Saúde (APS), enquanto membro da equipe multiprofissional, destaca-se, no presente estudo, o cuidado pré-natal, para o qual a enfermeira é legalmente respaldada e deve ter competência para desenvolvê-lo ${ }^{(1)}$. Para isso, depende da identificação correta das necessidades de saúde das mulheres, com consequente prestação de cuidados apropriados. Durante a sua prática, cabe à enfermeira desenvolver o cuidado junto à gestante e seu acompanhante com vistas a identificar possíveis injúrias maternas ou fetais que possam comprometer uma experiência positiva de gestação ou levar a um desfecho desfavorável da gravidez ${ }^{(1-2)}$.

A Organização Mundial da Saúde (OMS) preconiza que o cuidado durante o pré-natal ultrapasse práticas de caráter curativo ou preventivo de agravos, e que inclua ainda o aconselhamento de hábitos saudáveis, o planejamento das gestações, e a identificação e apoio a mulheres que se encontram em situação de violência perpetrada por seus parceiros ${ }^{(2)}$. Para isso, é essencial a presença de profissionais capacitados e com competência, que qualifiquem a atenção à saúde ${ }^{(3)}$.

Destaca-se que, ainda que hajam documentos ministeriais que apresentem competências da enfermeira, no sentido de atribuições, e outros que preveem competências essenciais ao exercício profissional, na especificidade da área obstétrica, como o da International Confederation of Midwives. Mas, neste estudo, parte-se da concepção de competência quanto à capacidade do sujeito em agir/atuar de modo eficaz, ao desenvolver uma ação quando se encontra em determinada situação em que necessite acionar os recursos que dispõem; porém, como cada situação é única, esses recursos não devem ser estanques ${ }^{(4)}$ para que possam responder às singularidades de cada situação. Dessa forma, a enfermeira, como profissional da APS, serviço preconizado como a porta de entrada ao Sistema Único de Saúde (SUS), pelas Redes de Atenção à Saúde (RAS) ${ }^{(1)}$, desenvolve um papel crucial, tendo o cuidado como objeto de seu trabalho, mediante ações de prevenção, promoção, reabilitação e tratamento à saúde ${ }^{(5)}$.
No cuidado à mulher, com o aprimoramento da competência de enfermeiras para o cuidado pré-natal, é possível alcançar não apenas melhores resultados na redução da morbimortalidade materna e fetal, mas também melhorias no cuidado, durante o processo de parturição ${ }^{(6)}$. Diante disso, suscitou-se a seguinte questão: quais as potencialidades, os entraves e as possibilidades quanto à competência de enfermeiras no cuidado pré-natal?

Com o intuito de fomentar a reflexão profissional, da gestão e do ensino quanto aos aspectos que poderiam ser aprimorados para alcançar um cuidado de maior competência, que valorize a profissão e responda às necessidades da população assistida, neste estudo, objetivouse identificar potencialidades, entraves e possibilidades quanto ao cuidado pré-natal com competência.

\section{MÉTODO}

Para responder ao objetivo do presente estudo, optou-se pela Revisão Integrativa da literatura, por ser um método que permite sintetizar informações de estudos publicados na bibliografia científica, possibilitando um levantamento do panorama atual, acerca da temática de interesse e indução de generalizações, bem como a identificação das lacunas existentes ${ }^{(7)}$.

Para o delineamento do estudo, seguiramse seis etapas ${ }^{(7)}$. Primeiramente, identificou-se o tema e elencou-se a seguinte questão de pesquisa ${ }^{(7)}$ : quais as potencialidades, os entraves e as possibilidades ao cuidado pré-natal com competência? Na segunda etapa, estabeleceramse os critérios para inclusão e exclusão dos estudos ${ }^{(7)}$. Conforme classificação quanto ao nível de evidência em: Nível I - revisão sistemática ou metanálise; Nível II - estudos controlados e aleatórios; Nível III - estudos controlados sem randomização; Nível IV - estudos caso-controle ou de coorte; Nível $V$ - revisão sistemática de estudos qualitativos ou descritivos; Nível VI estudos qualitativos ou descritivos e Nível VII opiniões ou $\operatorname{consensos}^{(8)}$, optou-se apenas por estudos originais, com níveis de evidência II, III, IV e VI, disponíveis na íntegra, nos idiomas inglês, espanhol ou português e que tivessem como objeto de estudo competência de enfermeiras, no cuidado pré-natal, na Atenção Primária à Saúde. Estudos de revisão, editoriais, opiniões/comentários, dissertações, teses, relatos de experiência e estudos reflexivos foram 
excluídos. Com o objetivo de abranger um maior quantitativo de publicações acerca da temática, optou-se por não delimitar o tempo de publicação dos artigos nas buscas.

Para o levantamento da literatura, foram consultadas, a partir do acesso ao portal de Periódicos CAPES/MEC, no mês de junho de 2018, as seguintes bases de dados de amplo acesso na área da saúde e enfermagem: Biblioteca Virtual de Saúde (BVS); National Library of Medicine
(PubMed/ Medline); Cumulative Index of Nursing and Allied Health Literature (CINAHL); Web of Science e a base multidisciplinar Scopus.

Para operacionalizar as buscas, realizou-se, inicialmente, uma consulta aos Descritores em Ciências da Saúde (DeCS) e termos (MeSH) Medical Subject Headings, da National Library, conformando as chaves de busca, conforme Figura 1.

Figura 1 - Estratégia de busca, segundo a base de dados, Curitiba, Paraná, Brasil, 2018.

\begin{tabular}{|l|l|}
\hline \multicolumn{1}{|c|}{ Base } & \multicolumn{1}{|c|}{ Combinação de busca } \\
\hline BVS & $\begin{array}{l}\text { Competence OR competency OR "professional competence" OR "nurse competence" OR "core } \\
\text { competencies" } A N D \text { pregnancy OR "antenatal care" OR "prenatal care" } A N D \text { nursing } O R \text { "obstetrical nursing" } \\
\text { OR "primary nursing care" OR "nursing care" }\end{array}$ \\
\hline $\begin{array}{l}\text { PubMed/ } \\
\text { Medline }\end{array}$ & $\begin{array}{l}\text { "Professional Competence" OR "Clinical Competence" OR "Social Skills" AND "Nurses" OR "Nurse Midwives" } \\
\text { Care" }\end{array}$ \\
\hline Web of Science & $\begin{array}{l}\text { "Professional competence" AND "prenatal care" AND "primary nursing care" AND "nurser practitioner" } \\
\text { OR "midwife/midwive" }\end{array}$ \\
\hline SCOPUS & $\begin{array}{l}\text { Competence OR competency OR "professional competence" OR "nurse competence" OR competencies AND } \\
\text { nursing OR midwifery AND "prenatal care" OR "antenatal care" OR pregnancy AND "primary health care" }\end{array}$ \\
\hline CINAHL & "Professional competence" AND nursing $A N D$ "prenatal care" \\
\hline
\end{tabular}

Fonte: As autoras, 2018.

$\mathrm{Na}$ busca inicial, foram identificados 2.208 estudos, nas cinco bases eletrônicas consultadas, seguiu-se aplicação dos filtros tipo de estudo, disponibilidade na íntegra e idiomas, conforme os critérios de inclusão, totalizando 1.036 artigos, dos quais procedeu-se à leitura do título, resumo, palavras-chave ou descritores. As pesquisas que não estavam em consonância com os critérios de inclusão foram desconsideradas, totalizando 191 artigos selecionados para leitura na íntegra. Após exclusão dos estudos duplicados e os que não atenderam ao escopo da revisão, foram incluídos para análise 17 estudos provenientes das bases de dados e três artigos encontrados em buscas complementares específicas, a partir das referências dos artigos consultados, totalizando 20 artigos, conforme processo descrito na Figura 2.

Figura 2 - Fluxograma do percurso para delimitação dos estudos com base no modelo PRISMA ${ }^{(9)}$ - Curitiba, PR, Brasil, 2018.

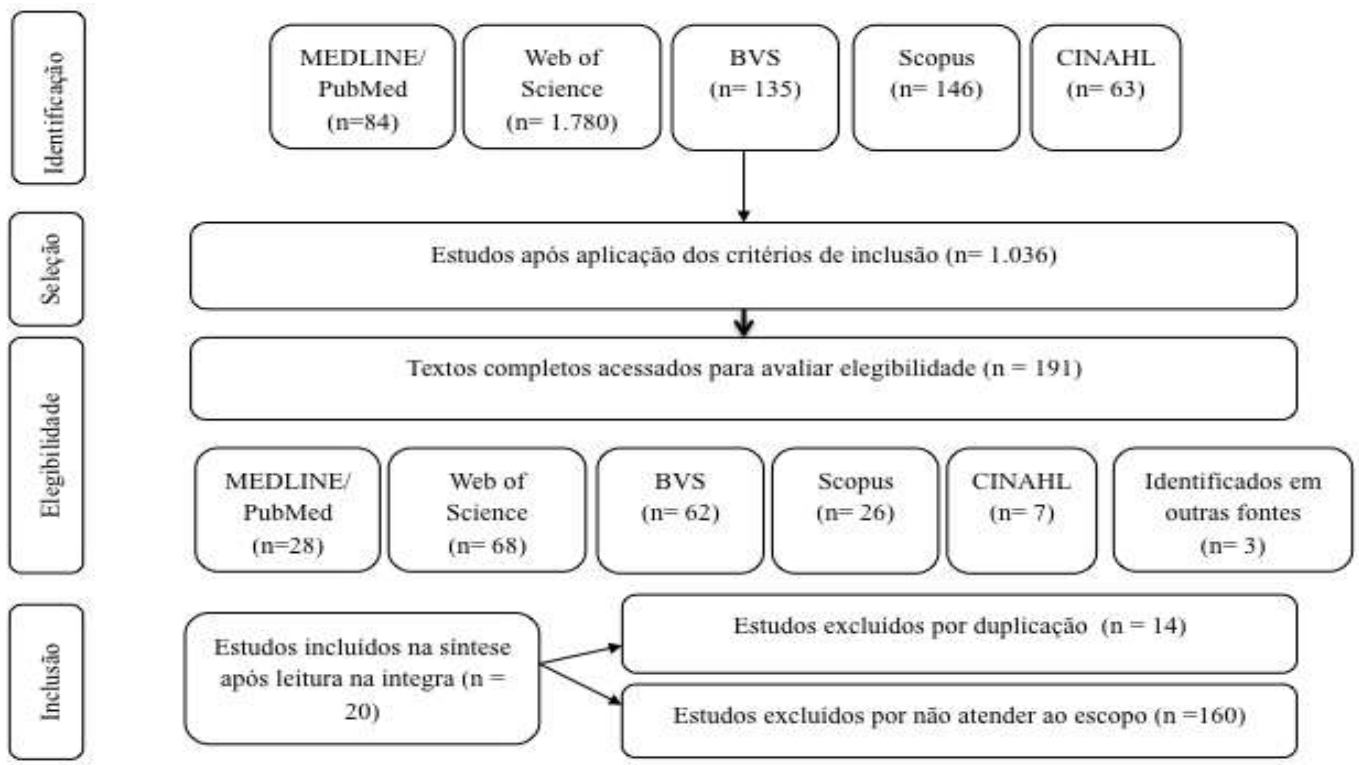

Fonte: As autoras, 2018 
$\mathrm{Na}$ terceira etapa, realizou-se a definição das informações a ser extraída dos estudos ${ }^{(7)}$ selecionados, mediante elaboração de instrumento contendo: título, autor, país e ano de publicação, base de dados, periódico, objetivo, delineamento e referencial metodológico e principais resultados apresentados pelo estudo. Após a organização dos estudos no instrumento estruturado, fez-se, na quarta etapa da revisão, a leitura, exploração e análise dos estudos ${ }^{(7)}$, os temas em comum que emergiram foram aglutinados quanto às potencialidades, entraves e possibilidades ao cuidado pré-natal com competência.

A quinta e sexta etapas da revisão consistiram na discussão e interpretação dos dados à luz da literatura e na apresentação da síntese do conhecimento ${ }^{(7)}$, respectivamente.

Os estudos incluídos na presente revisão foram publicados em periódicos de amplo acesso, não requerem sigilo ético, sendo desnecessária a apreciação pelo Comitê de Ética em Pesquisa (CEP). Entretanto, o presente estudo está contemplado em um projeto maior, aprovado sob Certificado de Apresentação para Apreciação Ética no 83070218.0.0000.0102.

\section{RESULTADOS E DISCUSSÃO}

Foram incluídos para análise 20 artigos apresentados, conforme Figura 3, todos classificados com nível de evidência VI, distribuídos em 18 periódicos, dos quais 11 (55\%) nacionais; sobressaíram as revistas Midwifery e Anna Nery, com dois artigos em cada.

O período de abrangência dos artigos foi de 2006 a 2018, sendo os anos de 2010 e 2012 com maior número de publicações. Houve predominância da abordagem quantitativa, com 11 (55\%) estudos, a abordagem qualitativa com sete (35\%) e os estudos de métodos mistos com dois (10\%) materiais selecionados.

A definição de competência foi identificada em quatro (20\%) artigos, os referenciais utilizados foram Ramos (2002) e Perrenoud (2001) com dois (10\%) estudos cada. Outros dois materiais mencionaram o embasamento nas Competências Essenciais para o exercício da Obstetrícia da International Confederation of Midwives (ICM), porém, não apresentaram definição. Os principais instrumentos utilizados para coleta dos dados foram: entrevista semiestruturada em metade dos estudos; questionários e formulários em oito (40\%) estudos, além da observação não participante em cinco (25\%), dos quais dois em concomitância com entrevistas semiestruturadas. Os profissionais de saúde foram os participantes de 18 (90\%) estudos, dos quais quatro (20\%) também participaram gestantes ou puérperas e acompanhantes e, em dois (10\%) foram consultadas apenas gestantes.

Figura 3 - Síntese de estudos selecionados. Curitiba, Paraná, Brasil, 2018.

\begin{tabular}{|c|c|c|c|}
\hline Título & $\begin{array}{l}\text { Ano/ } \\
\text { País }\end{array}$ & $\begin{array}{l}\text { Abordagem/ } \\
\text { Participantes }\end{array}$ & Principais resultados \\
\hline $\begin{array}{l}\text { Prenatal care: difficulties } \\
\text { experienced by nurses }\end{array}$ & $\begin{array}{l}2006 \\
\text { Brasil }\end{array}$ & $\begin{array}{c}\text { Quantitativa / } \\
\text { Enfermeiras }\end{array}$ & $\begin{array}{l}\text { Déficit de conhecimentos e habilidades profissionais, } \\
\text { falhas na graduação quanto ao cuidado pré-natal. É } \\
\text { preciso desenvolver habilidades (saber-fazer) que, } \\
\text { necessitam de conhecimento (saber) para existirem } \\
\text { atitudes (saber-ser). }\end{array}$ \\
\hline $\begin{array}{l}\text { Training of healthcare } \\
\text { personnel to improve } \\
\text { performance of community- } \\
\text { based antenatal care } \\
\text { program }^{(11)}\end{array}$ & $\begin{array}{c}2007 \\
\text { Paraguai }\end{array}$ & $\begin{array}{l}\text { Quantitativa / } \\
\text { Equipe de } \\
\text { Enfermagem }\end{array}$ & $\begin{array}{l}\text { Incrementou-se o conhecimento profissional e adesão } \\
\text { das gestantes ao pré-natal. A formação contínua e } \\
\text { supervisão contribuíram para o desenvolvimento } \\
\text { profissional e a } \\
\text { prática dos conhecimentos e habilidades adquiridos. }\end{array}$ \\
\hline $\begin{array}{l}\text { A percepção das enfermeiras } \\
\text { sobre a competência social } \\
\text { no desenvolvimento da } \\
\text { assistência pré-natal }^{(12)}\end{array}$ & $\begin{array}{l}2008 \\
\text { Brasil }\end{array}$ & $\begin{array}{l}\text { Qualitativa / } \\
\text { Enfermeiras }\end{array}$ & $\begin{array}{l}\text { Atualização e especialização deficitárias, múltiplos } \\
\text { vínculos empregatícios. Identificam a insuficiência do } \\
\text { modelo biomédico. Para melhorar a assistência, o } \\
\text { reconhecimento e a motivação institucional são } \\
\text { determinantes. }\end{array}$ \\
\hline $\begin{array}{l}\text { Assistência pré-natal: } \\
\text { competências essenciais } \\
\text { desempenhadas por } \\
\text { enfermeiros }^{(13)}\end{array}$ & $\begin{array}{l}2009 \\
\text { Brasil }\end{array}$ & $\begin{array}{l}\text { Quantitativa / } \\
\text { Enfermeiras e } \\
\text { Gestantes }\end{array}$ & $\begin{array}{l}\text { Baixa especialização na área. Exame físico da gestante } \\
\text { deficitário. É preciso que os profissionais compreendam } \\
\text { a importância da incorporação dos protocolos } \\
\text { assistencias e qualificação. }\end{array}$ \\
\hline
\end{tabular}




\begin{tabular}{|c|c|c|c|}
\hline Título & $\begin{array}{l}\text { Ano/ } \\
\text { País }\end{array}$ & $\begin{array}{l}\text { Abordagem/ } \\
\text { Participantes }\end{array}$ & Principais resultados \\
\hline $\begin{array}{l}\text { Maternity care and birth } \\
\text { preparedness in rural } \\
\text { Kyrgyzstan and Tajikistan }\end{array}$ & $\begin{array}{l}2010 \\
\text { Quirguistão } \\
\text { Tajiquistão }\end{array}$ & $\begin{array}{l}\text { Quantitativa / } \\
\text { Profissionais de } \\
\text { saúde, Gestantes e } \\
\text { acompanhantes }\end{array}$ & $\begin{array}{l}\text { Gestantes, acompanhantes e profissionais desconhecem } \\
\text { as complicações, durante a gravidez, parto e puerpério. } \\
\text { Indicam-se cursos preparatórios a mulheres e } \\
\text { acompanhantes, além do treinamento profissional. }\end{array}$ \\
\hline $\begin{array}{l}\text { Análise do exercício de } \\
\text { competências dos não } \\
\text { médicos para atenção à } \\
\text { maternidade }\end{array}$ & $\begin{array}{l}2010 \\
\text { Brasil }\end{array}$ & $\begin{array}{l}\text { Quantitativa / } \\
\text { Enfermeiras }\end{array}$ & $\begin{array}{l}\text { Déficit no exame físico-ginecológico, avaliação } \\
\text { nutricional e educação em saúde na visita domiciliar. Há } \\
\text { entraves pessoais e institucionais. É preciso capacitar, } \\
\text { valorizar e motivar o profissional para o cuidado. }\end{array}$ \\
\hline $\begin{array}{l}\text { Prenatal care by nurses in the } \\
\text { East Zone of the city of São } \\
\text { Paulo - Brazil }\end{array}$ & $\begin{array}{l}2010 \\
\text { Brasil }\end{array}$ & $\begin{array}{l}\text { Quantitativa / } \\
\text { Enfermeiras }\end{array}$ & $\begin{array}{l}\text { Desconhecimento e subvalorização são entraves ao } \\
\text { exercício das competências. Faz-se necessária a } \\
\text { valorização, capacitação profissional e parceria com } \\
\text { mulheres e famílias. }\end{array}$ \\
\hline $\begin{array}{c}\text { Exercise of essential } \\
\text { competencies for midwifery } \\
\text { care by nurses in São Paulo, } \\
\text { Brazil }^{(17)}\end{array}$ & $\begin{array}{l}2011 \\
\text { Brasil }\end{array}$ & $\begin{array}{l}\text { Quantitativa / } \\
\text { Enfermeiras }\end{array}$ & $\begin{array}{l}\text { Barreira do modelo de cuidado que considera a posição } \\
\text { hierárquica em detrimento da competência profissional } \\
\text { e prática baseada em evidências. Valorização e } \\
\text { motivação profissional promovem o cuidar centrado na } \\
\text { mulher. }\end{array}$ \\
\hline $\begin{array}{l}\text { O pré-natal realizado pelo } \\
\text { enfermeiro: a satisfação das } \\
\text { gestantes }\end{array}$ & $\begin{array}{l}2011 \\
\text { Brasil }\end{array}$ & $\begin{array}{l}\text { Qualitativa / } \\
\text { Gestantes }\end{array}$ & $\begin{array}{l}\text { Aprovação do pré-natal realizado pelo enfermeiro, } \\
\text { satisfação das gestantes quanto à competência na } \\
\text { completude da consulta, humanização e escuta ativa, } \\
\text { paciência para orientação em saúde diante das dúvidas } \\
\text { apresentadas. }\end{array}$ \\
\hline $\begin{array}{l}\text { O cuidado pré-natal na } \\
\text { Atenção Básica de Saúde sob } \\
\text { o olhar de gestantes e } \\
\text { enfermeiros }^{(19)}\end{array}$ & $\begin{array}{l}2012 \\
\text { Brasil }\end{array}$ & $\begin{array}{l}\text { Qualitativa / } \\
\text { Enfermeiras e } \\
\text { Gestantes }\end{array}$ & $\begin{array}{l}\text { Pré-natal necessita ultrapassar o âmbito tecnológico, } \\
\text { abranger aspectos humanísticos e atenção integral. } \\
\text { Escuta ativa para identificar necessidades de gestantes, } \\
\text { para atuar de maneira a respondê-las. }\end{array}$ \\
\hline $\begin{array}{l}\text { Cuidado Pré-natal às } \\
\text { adolescentes: competências } \\
\text { das enfermeiras }\end{array}$ & $\begin{array}{l}2012 \\
\text { Brasil }\end{array}$ & $\begin{array}{l}\text { Qualitativa / } \\
\text { Enfermeiras }\end{array}$ & $\begin{array}{l}\text { Abordagem dialógica para reconhecer necessidades de } \\
\text { gestantes adolescentes. Faz-se necessário parcerias além } \\
\text { dos serviços de saúde, treinamento e educação } \\
\text { continuada, associados a construção de protocolos } \\
\text { específicos. }\end{array}$ \\
\hline $\begin{array}{l}\text { Estudo das competências } \\
\text { essenciais na atenção pré- } \\
\text { natal: ações da equipe de } \\
\text { enfermagem em Cuiabá, } \\
\qquad \mathrm{MT}^{(21)}\end{array}$ & $\begin{array}{l}2012 \\
\text { Brasil }\end{array}$ & $\begin{array}{l}\text { Quantitativa / } \\
\text { Equipe de } \\
\text { Enfermagem }\end{array}$ & $\begin{array}{l}\text { Experiência pela prática profissional. Déficit no exame } \\
\text { físico, orientações e encaminhamentos. Ausência de } \\
\text { grupo de gestante e classificação de risco. Ações não } \\
\text { padronizadas ou com base em evidências. }\end{array}$ \\
\hline $\begin{array}{l}\text { Ações do pré-natal realizadas } \\
\text { pela equipe de enfermagem } \\
\text { na Atenção Primária à Saúde, } \\
\text { Cuiabá(22) }\end{array}$ & $\begin{array}{l}2013 \\
\text { Brasil }\end{array}$ & $\begin{array}{l}\text { Quantitativa / } \\
\text { Equipe de } \\
\text { Enfermagem }\end{array}$ & $\begin{array}{l}\text { Competências essenciais despadronizadas, déficit em } \\
\text { orientações e registros. Protocolos, parcerias com } \\
\text { associações de classe e instituições de ensino para o } \\
\text { aprimoramento profissional e com base em } \\
\text { competências. }\end{array}$ \\
\hline $\begin{array}{l}\text { Poor quality of antenatal care } \\
\text { services-Is lack of } \\
\text { competence and support the } \\
\text { reason? An observational and } \\
\text { interview study in rural areas } \\
\text { of Lao PDR }\end{array}$ & $\begin{array}{l}2013 \\
\text { Suécia }\end{array}$ & $\begin{array}{l}\text { Métodos mistos / } \\
\text { Enfermeiras }\end{array}$ & $\begin{array}{l}\text { Qualidade e desempenho profissional deficientes. Falta } \\
\text { de rotina e apoio, escassez de equipamentos, e } \\
\text { profissionais com competência. Suprir equipamentos } \\
\text { básicos, prover profissionais qualificados, treinamento } \\
\text { em serviço e protocolos são necessários para aprimorar } \\
\text { o conhecimento e as habilidades. }\end{array}$ \\
\hline $\begin{array}{l}\text { Primary healthcare worker } \\
\text { knowledge related to } \\
\text { prenatal and immediate } \\
\text { newborn care: a cross } \\
\text { sectional study in Masindi, } \\
\text { Uganda }{ }^{(24)} \\
\end{array}$ & $\begin{array}{l}2014 \\
\text { Uganda }\end{array}$ & $\begin{array}{l}\text { Quantitativa / } \\
\text { Enfermeiras, } \\
\text { Obstetrizes e } \\
\text { Assistentes de } \\
\text { enfermagem }\end{array}$ & $\begin{array}{l}\text { Conhecimento deficitário. Melhores resultados foram de } \\
\text { profissionais com mais de seis anos de atuação e, em } \\
\text { serviços de atenção primária, quando comparados aos } \\
\text { hospitalares. Há diferenças formativas entre os } \\
\text { profissionais e a necessidade de aprimorar os } \\
\text { conhecimentos. }\end{array}$ \\
\hline $\begin{array}{c}\text { Prenatal care: essential } \\
\text { actions developed by } \\
\text { nurses }^{(25)}\end{array}$ & $\begin{array}{l}2015 \\
\text { Brasil }\end{array}$ & $\begin{array}{l}\text { Quantitativa / } \\
\text { Enfermeiras }\end{array}$ & $\begin{array}{l}\text { Procedimentos e exames de rotina satisfatórios. } \\
\text { Qualificação profissional para ampliar o aspecto clínico, } \\
\text { avaliações do impacto pré-natal da enfermeira na } \\
\text { morbimortalidade materna e neonatal são necessários. }\end{array}$ \\
\hline $\begin{array}{l}\text { Facilitators of prenatal care in } \\
\text { an exemplar urban clinic }\end{array}$ & $\begin{array}{l}2016 \\
\text { EUA }\end{array}$ & $\begin{array}{l}\text { Qualitativa / } \\
\text { Gestantes }\end{array}$ & $\begin{array}{l}\text { Gestantes imigrantes ou de baixa renda valorizam } \\
\text { profissionais competentes, cuidado humanizado e } \\
\text { empático, consistentes a suas crenças culturais, o que } \\
\text { contribui à melhora dos resultados maternos. }\end{array}$ \\
\hline
\end{tabular}




\begin{tabular}{|c|c|c|c|}
\hline Título & $\begin{array}{l}\text { Ano/ } \\
\text { País }\end{array}$ & $\begin{array}{l}\text { Abordagem/ } \\
\text { Participantes }\end{array}$ & Principais resultados \\
\hline $\begin{array}{l}\text { Low-risk antenatal care: } \\
\text { family health strategy nurses' } \\
\text { atitudes }\end{array}$ & $\begin{array}{l}2016 \\
\text { Brasil }\end{array}$ & $\begin{array}{l}\text { Métodos mistos / } \\
\text { Enfermeiras }\end{array}$ & $\begin{array}{l}\text { Há dinamismo e pró-atividade profissionais. Entraves de } \\
\text { cunho pessoal e institucional. Educação em serviço e } \\
\text { aproximação academia-serviço para a qualificação e o } \\
\text { desenvolvimento de competências. }\end{array}$ \\
\hline $\begin{array}{l}\text { Woman-centred care during } \\
\text { pregnancy and birth in } \\
\text { Ireland: thematic analysis of } \\
\text { women's and clinician's } \\
\quad \text { experiences }{ }^{(28)}\end{array}$ & $\begin{array}{l}2017 \\
\text { Irlanda }\end{array}$ & $\begin{array}{l}\text { Qualitativa / } \\
\text { Puérperas, } \\
\text { Obstetrizes, } \\
\text { Médicos e } \\
\text { Enfermeiras }\end{array}$ & $\begin{array}{l}\text { Apoio governamental e conscientização profissional para } \\
\text { superação do modelo atual. Precariedade das relações } \\
\text { de trabalho e sobrecarga evidenciam que, mesmo } \\
\text { conhecendo modelos alternativos, profissionais não } \\
\text { implementam em sua prática. }\end{array}$ \\
\hline $\begin{array}{l}\text { Práticas sociais de } \\
\text { medicalização \& } \\
\text { humanização no cuidado de } \\
\text { mulheres na gestação }\end{array}$ & $\begin{array}{l}2018 \\
\text { Brasil }\end{array}$ & $\begin{array}{l}\text { Qualitativa / } \\
\text { Enfermeiras, } \\
\text { Médicos e } \\
\text { Dentistas }\end{array}$ & $\begin{array}{l}\text { Rotatividade de profissionais, precariedade de vínculos } \\
\text { empregatícios e sobrecarga de trabalho, com acúmulo } \\
\text { de funções, são entraves ao modelo humanizado. } \\
\text { Necessita-se remodelação de conceitos de saúde e } \\
\text { doença para os processos de trabalho. }\end{array}$ \\
\hline
\end{tabular}

Fonte: Dados obtidos do estudo, 2018.

A partir das informações coletadas dos artigos selecionados, elaborou-se um quadro síntese quanto às potencialidades do cuidado pré-natal desenvolvido por enfermeiras com competência, os entraves ao cuidado pré-natal com competência e as possibilidades para um cuidado pré-natal com competência (Figura 4).

Figura 4 - Potencialidades, entraves e possibilidades ao cuidado pré-natal com competência. Curitiba, Paraná, Brasil, 2018.

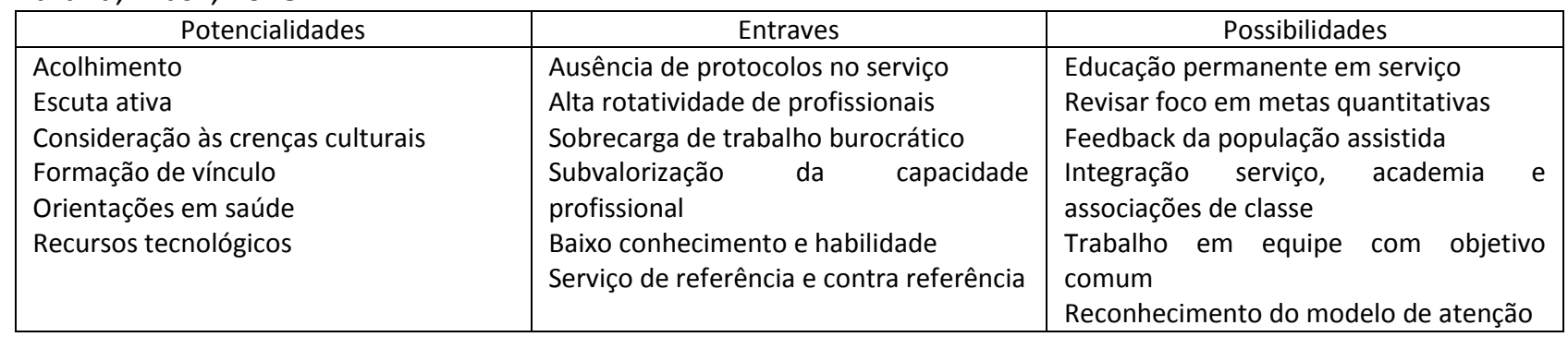

Fonte: As autoras, 2018.

\section{Potencialidades do cuidado pré-natal com competência}

Essa categoria foi conformada por sete estudos e apresenta o potencial do cuidado prénatal prestado por enfermeira com competência tanto no ponto de vista das gestantes que recebem o cuidado quanto dos profissionais que o realizam, sendo o período pré-natal destacado como um momento central para desenvolver cuidados que visam a garantir uma gravidez segura ${ }^{(25)}$.

O cuidado humanizado desenvolvido por profissionais competentes é valorizado pelas gestantes $^{(18,26)}$, especialmente pela capacidade de atenção às suas queixas, paciência e dedicação a orientações em saúde apresentados pelas enfermeiras $^{(18)}$. A presença de recursos tecnológicos e atenção aos aspectos biológicos também é apreciada pelas gestantes, contudo, evidencia-se a representatividade de cuidados que independem da disponibilidade de recursos materiais, como o acolhimento ${ }^{(18-19)}$, consideração das crenças culturais da gestante e família ${ }^{(26)}$, disponibilidade para sanar dúvidas e assiduidade do enfermeiro nas consultas ${ }^{(18-19,26)}$. Ou seja, a satisfação das gestantes quanto à consulta realizada pela enfermeira abrange, além da qualidade prestada quanto a práticas protocolares, mas em ações que favoreçam a formação do vínculo, envolvendo maiores componentes relacionais como a escuta ativa, o acolhimento, atenção às dúvidas, empatia ${ }^{(18-19)}$, apontados como facilitadores ao cuidado prénatal ${ }^{(30)}$.

Na perspectiva das enfermeiras, o cuidado pré-natal de qualidade foi compreendido como aquele com acolhimento, educação em saúde, atenção integral à gestante, número mínimo de seis consultas, serviço de referência e contrarreferência, além de trabalho em equipe ${ }^{(15-}$ $16,20,27)$. As enfermeiras identificam-se como profissionais proativas, que desenvolvem 0 acolhimento, buscando construção de vínculo para fortalecer a relação profissional-gestante, utilizando-se do diálogo como ferramenta para estabelecer o vínculo, bem como a escuta 
$\operatorname{ativa}^{(27)}$, sendo reconhecidas pela população como as profissionais que mais exercem a escuta $\operatorname{ativa}^{(12,18)}$

Diante do modelo biomédico preponderante na atenção à saúde, é mister a assimilação por parte das enfermeiras quanto a sua ineficiência para responder as necessidades das gestantes em sua integralidade ${ }^{(12)}$, mediante uma abordagem dialógica no cuidado, ${ }^{(20)}$ buscando avançar quanto à própria prática. Esse reconhecimento quanto a insuficiência do modelo atual de atenção à saúde é uma possibilidade para sua transformação ${ }^{(12,17)}$, uma vez que, desnaturalizar um problema corresponde à movimentação para buscar compreendê-lo e solucioná-lo. Como, por exemplo, ao analisar o aumento da cobertura da atenção pré-natal, que não representou expressiva melhoria da qualidade diante da desproporcional redução da mortalidade materna e infantil em vista dos esforços para impactar nesses índices ${ }^{(31)}$.

Dessa maneira, identifica-se que a competência profissional na enfermagem está diretamente relacionada com a melhoria da qualidade do atendimento prestado e da saúde pública ${ }^{(32)}$. Quanto ao pré-natal, que representa um momento primordial de cuidado à gestante $\mathrm{e}$ acompanhante, para desenvolvê-lo com competência e pautado pelos princípios da humanização, a enfermeira deve ampliar sua prática apenas ao biológico, abrangendo o contexto em que a gestante está inserida, utilizando-se do vinculo, escuta ativa e os demais recursos acima abordados para reconhecer necessidades e desenvolver sua prática profissional de modo a respondê-las ${ }^{(26,33)}$.

\section{Entraves ao cuidado pré-natal com competência}

Os 13 estudos que compuseram a presente categoria apontam limitações à prestação de um cuidado pré-natal com competência, destacandose a predominância de estudos quantitativos na literatura consultada (55\%). Com isso, percebe-se que as análises sobre a competência profissional ainda estão tradicionalmente centradas na aquisição de conhecimentos e técnicas e/ou no desempenho individual dos trabalhadores ${ }^{(34)}$. É preciso refletir sobre qual prisma a competência será considerada diante da evidenciação da capacitação em serviço como uma estratégia para desenvolvê-la, uma vez que, não se trata apenas do acúmulo de conhecimentos, mas sim da forma como o profissional mobiliza, de maneira racional e reflexiva, os recursos disponíveis em determinada situação ${ }^{(4)}$.

As pesquisas que verificaram o cuidado pré-natal sob o prisma das Competências Essenciais para o Exercício da Obstetrícia da ICM $(10,15-16,24)$, identificaram inconformidades ao preconizado pelo documento. As dificuldades para desenvolver um cuidado com competência abrangem questões relativas à limitações pessoais do profissional e institucionais ${ }^{(15-17,28-29)}$. Como barreiras do serviço tem-se a falta de protocolos que direcionem o cuidado pré-natal ${ }^{(15-}$ 16), que, quando associados a rotinas padronizadas e formações direcionadas à atenção possibilitam ampliar o conhecimento dos enfermeiros e a sua adesão para a realização do pré-natal $^{(21)}$. Somando-se a isso, apontam o reduzido quantitativo e a rotatividade dos profissionais, que também dificultam a continuidade do cuidado, atrelado à sobrecarga de trabalho, em razão do acúmulo de funções burocráticas, desvalorização da capacidade e competência das enfermeiras ${ }^{(15,28-29)}$, confirmam condições que corroboram para o enfoque da prática reduzir-se ao cumprimento de tarefas ${ }^{(29)}$ direcionadas ao monitoramento do desenvolvimento fisiológico da gestação.

Como barreiras pessoais encontram-se a falta de conhecimento ${ }^{(10,13,15-17,24)}$, e de habilidade dos profissionais que realizam o pré-natal ${ }^{(10,23)}$, além da precariedade de vínculos empregatícios, com baixa remuneração ${ }^{(12,15,28-29)}$, fazendo com que muitos profissionais tenham jornadas duplas ou triplas de trabalho. Ademais, observa-se uma dificuldade na sedimentação do vínculo enfermeira-gestante-acompanhante, essencial para alcançar maior profundidade no reconhecimento de necessidades das gestantes e extrapolar o âmbito biológico ${ }^{(25)}$, uma vez que, essa aproximação demanda dedicação e tempo, dificultados pela demanda do serviço quanto ao tempo disponível ${ }^{(23)}$.

O desconhecimento do potencial da enfermeira pelos sistemas de saúde, insuficiência de capacitações ou educação permanente ${ }^{(10,14,16-}$ 17,23-24), os conflitos e rivalidades que enfrentam com a classe médica, falta de união e fortalecimento profissional, e, principalmente, ao não estabelecimento de parcerias com as mulheres, a fim de gozar de maior reconhecimento ${ }^{(15-16)}$, também são entraves. A limitação dos enfermeiros quanto à solicitação de determinados exames, durante o pré-natal e a demora nos resultados, ausência de referência e 
contrarreferência, carência de recursos materiais, e falta de trabalho em equipe são apontados como fatores dificultantes ao cuidado competente ${ }^{(23)}$.

Quanto às gestantes, a insatisfação perpassa a ausência de referência e contrarreferência entre os serviços de saúde e a carência de informações ${ }^{(19)}$. $O$ atendimento meramente protocolar, precedido de longas esperas para abordagem de questões pontuais e protocolares, conformam barreiras para adesão ao pré-natal ${ }^{(30)}$, e a prática de educação em saúde confere maior qualidade ao cuidado prestado ${ }^{(19)}$. Contudo, é preciso que esses profissionais tenham competência para que essas orientações não sejam apenas a transmissão de informações ${ }^{(25)}$, mas que efetivem um cuidado à gestante em sua integralidade, a partir do contexto e das necessidades da gestante, para que a orientação realizada faça sentido e incida sobre sua realidade.

Um cuidado que restringe o olhar às necessidades de saúde da gestante faz-nos questionar quanto a adequabilidade do pré-natal comparando-se a sua continuidade. Estudo mexicano identificou uma proporção de $98,4 \%$ de cobertura de consultas realizadas por mulheres durante a ultima gestação, no entanto, a adequabilidade esteve presente em apenas $71,5 \%$ dos casos, apresentando melhor adequação entre mulheres com maior poder aquisitivo e escolaridade ${ }^{(35)}$.

Em consonância, estudo nacional identificou baixa adequabilidade da atenção prénatal (15\%), principalmente quanto à completude do exame físico, recebimento de orientações e realização de todos os exames complementares. Mulheres com maior renda, residentes em cidades com maior Índice de Desenvolvimento Humano (IDH) e localizadas na Região Sudeste receberam atenção pré-natal mais adequada ${ }^{(36)}$. Salienta-se a relevância de um cuidado desenvolvido por profissionais com competência para identificar as necessidades das gestantes e incidir sobre estas, buscando a equidade na atenção à saúde ${ }^{(36)}$, primando pela essência da profissão, que é o cuidado.

\section{Possibilidades para desenvolvimento e manutenção de competência no cuidado pré- natal}

Na presente categoria, conformada por 16 estudos, são apresentadas possibilidades para desenvolver a competência de enfermeiras no cuidado pré-natal e promover a sua manutenção. A educação permanente em serviço e maior oferta de capacitação profissional são apontadas pelos estudos como cruciais para desenvolver competência para um cuidado pré-natal de qualidade com vista à maternidade segura ${ }^{(11-}$ $14,16,20,22-25,27)$

Para que as mulheres tenham uma experiência positiva da gravidez, é preciso que as ações de atenção à saúde sejam direcionadas tanto à manutenção da normalidade física quanto também sociocultural e de parâmetros saudáveis à mulher e ao bebê, para uma transição ao parto e nascimento como um evento positivo ${ }^{(37)}$. Sendo a enfermeira e demais profissionais da saúde mediadores, desse processo numa atuação conjunta ${ }^{(3)}$, destaca-se o papel daquela quanto a sua representatividade no sistema de saúde, respaldo legal ao cuidado com autonomia e na formação de opinião junto a sociedade civil. Salienta-se a necessidade de investimentos em sua formação e capacitação para o cuidado, de modo que possibilite a interligação entre os conhecimentos, habilidades e atitudes ${ }^{(38)}$.

Para efetivação de um cuidado individualizado, conforme a necessidade da gestante, baseado em evidências e na continuidade das ações ${ }^{(39)}$, é preciso um serviço diferenciado, com profissionais habilitados a atuar a partir das boas práticas clínicas, com o fornecimento de informações relevantes e oportunas, além do apoio psicossocial e emocional ${ }^{(37)}$. Sendo a educação permanente em serviço, evidenciada como uma potente ferramenta para o desenvolvimento da competência de enfermeiras no cuidado prénatal ${ }^{(12-14,16,19-20,22-23,27)}$, principalmente, diante da necessidade de conhecimentos que o profissional precisa dispor para agir em determinada situação, uma vez que, as situações são mutáveis, bem como os conhecimentos e recursos necessários e, por consequência, a competência profissional também modifica-se ${ }^{(4)}$, necessitando de constante aprimoramento.

O melhoramento da integração do sistema de referência e contrarreferência dos serviços é destacado como ponto positivo para fomentar a manutenção de competências ${ }^{(16)}$. Há necessidade de reconhecimento e motivação profissional por parte da instituição ${ }^{(12,15-17,23)}$, não apenas a valorização da dimensão tecnológica nas práticas de saúde, refletida na execução de tarefas e o cumprimento de metas quantitativas ${ }^{(12)}$. Faz-se 
necessário fomentar o trabalho em rede, para efetivação da proposta das Redes de Atenção à Saúde como a porta de entrada ao SUS, com vista a melhorar o serviço de referência e contrarreferência, além do trabalho em equipe $^{(19)}$. Salienta-se que, para estarem alinhados num trabalho que tenha uma mesma finalidade, é necessário que desenvolvam competências coletivas, de modo que as ações entre os profissionais se articulem, não se justaponham umas às outras ${ }^{(4)}$.

Diante disso, evidencia-se a relevância da participação da instituição para efetivação de propostas que visem ao desenvolvimento de competência, mediante a definição de suas diretrizes organizacionais e atribuições consoantes "a agregação de valor social ao indivíduo e econômico à instituição" ${ }^{(40)}$. Para que se efetivem os princípios da humanização preconizados aos serviços de saúde, os profissionais devem pactuar desses princípios, a partir de uma reforma do processo de trabalho e remodelação dos conceitos de saúde e doença ${ }^{(29)}$, de modo que, todos os profissionais estejam alinhados em prol de um objetivo em comum centrado em responder às necessidades da gestante $e^{(12,17)}$.

Outra possibilidade para o desenvolvimento e manutenção da competência, no cuidado pré-natal, concerne ao feedback da população assistida e o seu reconhecimento acerca do cuidado recebido, que também fortalecem os profissionais e favorecem uma prática com competência ${ }^{(12)}$. Dessa forma, é necessária a manutenção contínua da competência profissional, sendo de responsabilidade, tanto do próprio indivíduo quanto da organização e das associações de classe $^{(6)}$, que podem articular-se no sentido de fortalecer a qualidade do serviço prestado e a própria profissão. Nesse caso, pode ser útil aplicar estratégias como fornecer suporte de gerenciamento adequado para os enfermeiros que permitam o crescimento profissional; avaliação justa das práticas desenvolvidas; senso de autonomia profissional e melhora nos recursos organizacionais ${ }^{(32)}$.

Para a manutenção dos conhecimentos e habilidades desenvolvidos, é necessário associar estratégias de supervisão contínua dessas ações ${ }^{(11)}$, além do desenvolvimento de protocolos e fomento à capacitação profissional, mediante cursos de especialização na área ${ }^{(13)}$. Ademais, o fornecimento de cursos a gestantes e acompanhantes, no sentido de conscientizá-los e inseri-los no cuidado, pode complementar o trabalho com competência da enfermeira para qualidade no pré-natal $^{(14)}$, podendo inclusive serem firmadas parcerias com outras instituições sociais para ampliar a abrangência das orientações e ações desenvolvidas ${ }^{(20)}$.

A fim de que todos esses aspectos sejam alcançados, é imprescindível o apoio e comprometimento governamental para alcançar modelos de cuidado com ênfase ao cuidado centrado na mulher ${ }^{(28)}$, bem como a parceria entre academia, serviço ${ }^{(15)}$ e associações de classe $^{(21-22,27)}$, e que a enfermeira estabeleça o vínculo com a gestante e acompanhante ${ }^{(16)}$. 0 fortalecimento entre serviço e instituição de ensino favorece, desde a formação, que estejam alinhados quanto á competência necessária no serviço para o cuidado pré-natal. Uma vez que, diante dos diferentes conceitos existentes, incorre-se em um descompasso da formação e da prática, em que o "como" ocorre a formação não converge ao "como" desenvolve-se à prática.

A multiplicidade de terminologias utilizadas para caracterizar a competência profissional, bem como a diversidades de conceituações para tal, podem ser consideradas limitações, neste estudo, uma vez que, os descritores foram direcionados especificamente ao termo competência, podendo não ter compreendido artigos que abordassem o tema mediante outros descritores.

Considerando-se que o pré-natal desenvolvido por enfermeira com competência contribui, além da melhora das metas quantitativas do serviço, para atender às necessidades da gestante em sua integralidade, refletindo não somente num pré-natal de qualidade, mas também na conscientização da mulher quanto ao processo de gestar e parir. Reconhecer essa lacuna, na literatura, quanto à prática da enfermeira, permite ao serviço estruturar-se quanto à necessidade de capacitação dos profissionais, bem como à academia reorganizar-se no processo ensinoaprendizagem para a formação de um profissional crítico e reflexivo sobre sua prática, e que seja norteada pelas melhores evidências para o cuidado.

\section{CONSIDERAÇÕES FINAIS}

Quanto às potencialidades do cuidado prénatal desenvolvido com competência, as perspectivas de gestantes e enfermeiras convergem quanto a necessidade de ser um 
espaço para educação em saúde, acolhimento e escuta ativa das queixas e necessidades da gestante. As profissionais identificam que $o$ modelo biomédico é insuficiente para responder a essas necessidades, e desenvolver um cuidado com competência possibilitaria ampliá-lo.

As limitações pessoais quanto a conhecimento e habilidades, associadas à organização do processo de trabalho com reduzido quantitativo de profissionais, alta rotatividade e sobrecarga de atividades burocráticas constituem entraves a um pré-natal que identifique e responda as necessidades da gestante e família, principalmente pela dificuldade de conformação do vínculo profissional-gestante-acompanhante.

Para superar esses entraves e fomentar as potencialidades, faz-se necessário tanto empenho pessoal em buscar aprimoramento, bem como é preciso que os serviços incorporem programas de educação permanente e contínuo monitoramento, partindo-se da identificação das lacunas dos profissionais para direcionar a estruturação de módulos que coadunem às necessidades apresentadas para desenvolver a competência das enfermeiras. Outras possibilidades que devem estar associadas, são a reorganização dos serviços para que a equipe trabalhe em prol de um objetivo comum, responder às necessidades da população, necessitando de esforços tanto em nível do próprio serviço, mas também das instituições formadoras e associações de classe.

\section{REFERÊNCIAS}

1- Brasil, Ministério da Saúde. Protocolos da atenção básica: Saúde das mulheres. Brasília: Ministério da Saúde; 2016.

2- World Health Organization (WHO). Recommendations on antenatal care for a positive pregnancy experience. Geneva: WHO; 2016.

3- Barbaro MC, Lettiere A, Nakano AMS. Prenatal care for adolescents and attributes of primary health care. Rev Latino-Am Enfermagem 2014;22(1):108-14. DOI: $\quad$ 10.1590/01041169.3035 .2390

4- Perrenoud P. Desenvolver competências ou ensinar saberes? A escola que prepara para a vida. Porto Alegre: Penso; 2013.

5- Organização Pan-Americana de Saúde (OPAS). Ampliação do papel dos enfermeiros na atenção primaria à saúde. Washington, D.C.: OPAS; 2018.
6- Casey M, Cooney A, O'Connel R, Hegarty J-M, Brady A-M, O'Reilly $P$, et al. Nurses', midwives' and key stakeholders' experiences and perceptions on requirements to demonstrate the maintenance of professional competence. J Adv Nurs. 2017;73(3):653-64. DOI:

10.1111/jan.13171

7- Mendes KDS, Silveira RCCP, Galvão CM. Revisão integrativa: Método de pesquisa para a incorporação de evidências na saúde e na enfermagem. Texto Contexto-Enferm. 2008;17(4):758-64. DOI: $10.1590 /$ S0104$\underline{07072008000400018}$

8- Souza MT, Silva MD, Carvalho R. Revisão integrativa: O que é e como fazer. Einstein 2010;8(1):102-6. DOI: $10.1590 /$ s167945082010rw1134

9- Moher D, Shamseer L, Clarke M, Ghersi D, Liberati A, Petticrew $P$, et al. Preferred reporting items for systematic review and meta-analysis protocols (PRISMA-P) 2015 statement. Syst Rev. 2015;4:1. DOI: 10.1371/journal.pmed.1000097

10- Dotto L, Moulin N, Mamede M. Prenatal care: Difficulties experienced by nurses. Rev Latino-Am Enfermagem 2006;14(5):682-8. DOI: 10.1590/S0104-11692006000500007

11- Ohnishi M, Nakamura K, Takano T. Training of healthcare personnel to improve performance of community-based antenatal care program. Adv Health Sci Educ Theory Pract. 2007;12(2):147-56. DOI: $10.1007 / \mathrm{s} 10459-005-2329-\mathrm{x}$

12- Lima YMS, Moura MAV. A percepção das enfermeiras sobre a competência social no desenvolvimento da assistência pré-natal. Esc Anna Nery. 2008;12(4):672-8. DOI: 10.1590/S1414-81452008000400010

13- Cunha MA, Mamede MV, Dotto LMG, Mamede FV. Assistência pré-natal: Competências essenciais desempenhadas por enfermeiros. Esc Anna Nery. 2009;13(1):145-53. DOI: 10.1590/S1414-81452009000100020

14- Wiegers TA, Boerma WG, Haan O. Maternity care and birth preparedness in rural Kyrgyzstan and Tajikistan. Sex Reprod Healthc. 2010;1(4):189-94. DOI:

10.1016/i.srhc.2010.08.004

15- Narchi NZ. Análise do exercício de competências dos não médicos para atenção à maternidade. Saúde Soc. 2010;19(1):147-58. DOI: 10.1590/S0104-12902010000100012

16- Narchi NZ. Prenatal care by nurses in the East Zone of the city of São Paulo - Brazil. Rev Esc Enferm USP. 2010;44(2):266-73. DOI: 10.1590/S0080-62342010000200004 
17- Narchi NZ. Exercise of essential competencies for midwifery care by nurses in São Paulo, Brazil. Midwifery. 2011;27(1):23-9. DOI: 10.1016/j.midw.2009.04.007

18- Barbosa TLA, Gomes LMX, Dias OV. O prénatal realizado pelo enfermeiro: A satisfação das gestantes. Cogitare Enferm. 2011;16(1):29-35. DOI: $10.5380 / c e . v 16 i 1.21108$

19- Guerreiro EM, Rodrigues DP, Silveira MAM, Lucena NBF. O cuidado pré-natal na atenção básica de saúde sob o olhar de gestantes e enfermeiros. REME, Rev Min Enferm. 2012 [citado em 15 jan 2019]; 16(3):315-23. Disponível em:

http://www.reme.org.br/artigo/detalhes/533 20- Parenti PW, Silva LCFP, Melo CRM, Clapis MJ. Cuidado pré-natal às adolescentes: Competências das enfermeiras. Rev Baiana Enferm. 2012;26(2):498-509. DOI: 10.18471/rbe.v26i2.6534

21- Duarte SJH, Mamede MV. Estudo das competências essenciais na atenção pré-natal: Ações da equipe de enfermagem em Cuiabá, MT. Enferm Foco. 2012;3(2):75-80. DOI: 10.21675/2357-707X.2012.v3.n2.259

22- Duarte SJH, Mamede MV. Ações do pré-natal realizadas pela equipe de enfermagem na atenção primária à saúde, Cuiabá. Cienc Enferm. 2013;19(1):117-29. DOI: $10.4067 /$ S071795532013000100011

23- Manithip C, Edin K, Sihavong A, Wahlström $\mathrm{R}$, Wessel H. Poor quality of antenatal care services-Is lack of competence and support the reason? An observational and interview study in rural areas of Lao PDR. Midwifery. 2013;29(3):195-202. DOI: 10.1016/j.midw.2011.12.010

24- Ayiasi RM, Criel B, Orach CG, Nabiwemba E, Kolsteren $P$. Primary healthcare worker knowledge related to prenatal and immediate newborn care: A cross sectional study in Masindi, Uganda. BMC Health Serv Res. 2014;14:65. DOI: 10.1186/1472-6963-14-65

25- Baptista R, Dutra M, Coura A, Sousa F. Prenatal care: Essential actions developed by nurses. Enferm Glob. 2015 [citado em 15 jan 2019]; 14(4):96-142. Disponível em: http://scielo.isciii.es/pdf/eg/v14n40/en clinica5. pdf

26- Phillippi JC, Holley SL, Payne K, Schorn MN, Karp SM. Facilitators of prenatal care in an exemplar urban clinic. Women Birth. 2016;29(2):160-7.

$\underline{10.1016 / \text { i.wombi.2015.09.007 }}$
27- Gonçalves MD, Kowalski ISG, Sá AC. Low-risk antenatal care: Family health strategy nurses' attitudes. Rev Enferm UERJ 2016;24(6):1-6. DOI: 10.12957/reuerj.2016.18736

28- Hunter A, Devane D, Houghton C, Grealish A, Tully A, Smith V. Woman-centred care during pregnancy and birth in Ireland: Thematic analysis of women's and clinicians experiences. BMC Pregnancy Childbirth 2017;17(1): 322. DOI: 0.1186/s12884-017-1521-3

29- Warmling CM, Fajardo AP, Meyer DE, Bedos C. Práticas sociais de medicalização \& humanização no cuidado de mulheres na gestação. Cad Saúde Pública 2018;34(4): 1-11. DOI: 10.1590/0102-311x00009917

30- Heaman MI, Sword W, Elliott L, Moffatt M, Helewa ME, Morris $H$, et al. Barriers and facilitators related to use of prenatal care by inner-city women: Perceptions of health care providers. BMC Pregnancy Childbirth 2015;15:2. DOI: 10.1186/s12884-015-0431-5

31- Nunes JT, Gomes KRO, Rodrigues MTP, Mascarenhas MDM. Qualidade da assistência pré-natal no Brasil: Revisão de artigos publicados de 2005 a 2015. Cad Saúde Coletiva 2016;24(2):252-61. DOI: 10.1590/1414$462 \times 201600020171$

32- Karami A, Farokhzadian J, Foroughameri G. Nurses' professional competency and organizational commitment: Is it important for human resource management? PLoS One 2017;12(11):e0187863. DOI: 10.1371/journal.pone. 0187863

33- Cardelli AAM, Marrero TL, Ferrari RAP, Martins JT, Serafim D. Expectations and satisfaction of pregnant women: Revealing prenatal care in primary care. Invest Educ Enferm. 2016;34(2):252-60. DOI: 10.17533/udea.iee.v34n2a04

34- Fullerton J, Ghérissi $A$, Johnson $P$, Thompson J. Competence and competency: Core concepts for international midwifery practice. Int J Childbirth. 2011;1(1):4-12. DOI: 10.1891/21565287.1.1.4

35- Hereda-Pi I, Servan-Mori E, Darney BG, Reyes-Morales $H$, Lozano R. Measuring the adequacy of antenatal health care: $A$ national cross-sectional study in Mexico. Bull World Health Org. 2016;94:452-61. DOI: 10.2471/BLT.15.168302

36- Tomasi E, Fernandes PAA, Fischer T, Siqueira FCV, Silveira DS, Thumé E, et al. Qualidade da atenção pré-natal na rede básica de saúde do Brasil: Indicadores e desigualdades sociais. Cad 
Saúde Pública 2017;33(3):1-11. DOI: $\underline{10.1590 / 0102-311 \times 00195815}$

37- Downe S, Finlayson K, Tunçalp Ö, Gülmezoglu AM. What matters to women: $A$ systematic scoping review to identify the processes and outcomes of antenatal care provision that are important to healthy pregnant women. BJOG 2016;123(4):529-39. DOI: 10.1111/1471-0528.13819

38- Melo WS, Oliveira PJF, Monteiro FPM, Santos FCA, Silva MJN, Calderon CJ, et al. Guide of attributes of the nurse's political competence: $A$ methodological study. Rev Bras Enferm. 2017;70(3):526-34. DOI: 10.1590/0034-71672016-0483

39- Kennedy HP, Cheyney M, Dahlen HG, Downe $\mathrm{S}$, Foureur MJ, Homer KSE, et al. Asking different questions: A call to action for research to improve the quality of care for every woman, every child. Birth. 2018;45(3):222-31. DOI: 10.1111/birt.12361

40- Kobayashi RM, Leite MM. Desenvolvendo competências profissionais dos enfermeiros em serviço. Rev Bras Enferm. 2010;63(2):243-9. DOI: $\underline{10.1590 / \mathrm{S} 0034-71672010000200012}$

Note: Este artigo é resultante do relatório de doutoramento intitulado "Competência de Enfermeiras no Cuidado Prénatal na Atenção Primária à Saúde", pelo Programa de PósGraduação em Enfermagem da Universidade Federal do Paraná.

Agradecimento à Coordenação de Aperfeiçoamento de Pessoal de Nível Superior - CAPES pelo financiamento de bolsa de Pós-Graduação.

Recebido em: 27/07/2019

Aprovado em: 10/12/2019

Endereço de correspondência:

Deisi Cristine Forlin Benedet

Rua Lamenha Lins, 260. Apto 1202 - Centro

CEP: 80250-020 - Curitiba/PR - Brasil

E-mail: deisiforlin@ufpr.br 